\title{
Fronteras simbólicas y redes intelectuales. Una propuesta
}

Artículo recibido: 09-12-2016 | Artículo aceptado: 22-02-2017

DOI: $10.25100 /$ hye.v13i49.5849

\section{Alexandra Pita}

Licenciada en Historia por la Universidad Nacional de Córdoba, Maestra y Doctora en Historia por El Colegio de México. Se desempeña como profesora titular y coordinadora de la Maestría en Historia de la Universidad de Colima. Recientemente ha publicado los artículos "Panamericanismo y nación. La perspectiva de Samuel Guy Inman”, Anuario IEHS, Instituto de Estudios Históricos y Sociales 32, 1, (2017): 135-154; y "Jaime Torres Bodet and the Intellectual/International Cooperation of Postwar” 8, 2, (2015). Correo electrónico: alepitag@ucol.mx

Forma de citar este artículo: Pita, Alexandra. "Fronteras simbólicas y redes intelectuales. Una propuesta”. Historia y Espacio, vol. 13, n’ 49 (2017): 39-62.

Artículo Tipo 2: de reflexión. Documento que presenta resultados de investigación desde una perspectiva analítica, interpretativa o crítica del autor, sobre un tema específico, recurriendo a fuentes originales. 


\section{Fronteras simbólicas y redes intelectuales. Una propuesta}

Resumen: Las redes intelectuales han sido poco estudiadas y menos aún se ha planteado de manera específica el significado de sus fronteras. Por ello, este ensayo realiza un breve balance historiográfico de algunos trabajos sobre las fronteras en la historia, el papel del intelectual como sujeto de frontera y el carácter simbólico de aquellas mencionadas en algunos trabajos de redes intelectuales. En una segunda parte, se enfoca en una red en particular, La Unión Latino Americana durante sus primeros años (1923-1925), para reflexionar sobre dos coyunturas específicas: la de su fundación, para observar cómo una nueva red se entrelaza con otras, y cuando surge una ruptura importante en ella y se separan algunos de sus miembros para formar otra red. Si en la primera la frontera es una zona porosa de libre tránsito, en la segunda se convierte en un límite infranqueable de exclusión. Palabras clave: fronteras simbólicas, redes intelectuales, mediadores culturales

\section{Symbolic Borders and Intellectual Networks. A Proposal}

Abstract: The intellectual networks have been little studied and even less has been raised specifically the significance of its borders. Therefore, this essay makes a brief historiographic balance of some work on the borders in the history, the role of the intellectual as a subject of border and the symbolic character of those mentioned in some works of intellectual networks. In a second part, focuses on a particular network, the Latin American Union during its early years (1923-1925), to reflect specifically on two important moments: the one of its foundation to observe how a new network intertwines with other and when an important rupture arises in it and some of its members are separated to form another network. If in the first the border is a porous zone of free transit in the second it becomes an impassable limit of exclusion.

Key words: symbolic borders, intelectual networks, Cultural mediators

\section{Fronteiras simbólicas e redes intelectuais. Uma proposta}

Resumo: As redes intelectuais têm sido pouco estudadas e ainda menos tem sido exposto de maneira específica o significado das suas fronteiras. Por conseguinte, este ensaio faz um breve balanço de alguns trabalhos historiográficos sobre as fronteiras na história, o trabalho do intelectual como parte de fronteira e o carácter simbólico de aqueles mencionados em alguns trabalhos de redes intelectuais.Numa segunda parte, enfoca-se numa rede em particular, A União Latino-Americana durante seus primeiros anos (1923-1925), para refletir especificamente sobre dois conjunturas específicas da sua fundação para observar como uma nova rede está entrelaçada com outras e quando surge um rompimento importante em ela e separam-se alguns do seus membros para formar outra rede. Se na primeira fronteira é uma zona porosa de livre trânsito na segunda torna-se num limite infranqueável de exclusão.

Palavras-chave: Fronteiras simbólico, Redes intelectuais, mediadores culturais. 


\section{Alexandra Pita \\ Fronteras simbólicas y redes intelectuales. Una propuesta}

\section{Introducción}

La frontera ha sido representada frecuentemente como una entidad casi concreta, otorgándole una materialidad que olvida en gran parte su esencia como construcción histórica. Sin embargo, al incorporar la variable del imaginario como objeto de estudio, las ciencias sociales han comenzado a estudiar los territorios nacionales y sus fronteras desde un punto de vista que busca desnaturalizar "los espacios de la soberanía estatal". Esto ha diluido la idea de fronteras naturales desde el relato geopolítico para comenzar a estudiarlas a partir de la "contingencia y la porosidad". Así, la frontera abandonó su materialidad para pasar a ser simbólica, como un límite de cultura e identidad que cambia, se multiplica y resignifica a través de la acción de los sujetos sociales ${ }^{1}$. Este giro interpretativo no implica que el concepto frontera haya perdido su capacidad de ser polisémico ni, por ende, conflictivo, puesto que depende del vínculo establecido entre "actores, discursos, procesos y formas de poder"2.

1 Alejandro Grimson, “Disputas sobre las fronteras”, en Teoría de la frontera. Los límites de la política cultural, eds. Scott Michaelsen y David Johnson (Barcelona: Gedisa, 2003), 13-15, 17.

2 Victoria Crespo define tres concepciones de frontera predominantes en la historia. Una primera, definida por el conflicto entre el ellos y el nosotros, en la que la frontera es un límite que divide irremediablemente perdiendo su porosidad y flexibilidad, y que se asocia a la unidad político territorial sobre la que se sostienen los estados modernos. Una segunda que, a diferencia de la anterior, concibe la frontera como un consenso donde se encuentran las partes para facilitar los intercambios a través de zonas de confluencia flexibles. Esto refiere a unidades político territoriales que no se limitan al Estado moderno porque implica un proceso y, por último, una tercera en la que los contornos son flexibles superando el Estado 
Desde el ámbito de la historia, las fronteras han sido un tema relevante para entender procesos complejos de construcción de los Estados Nacionales. En América Latina, en particular, hay muchos ejemplos de este tipo de trabajos y, desde una mirada renovada de la historia política y social, se ha replanteado el valor de lo simbólico al pensar las fronteras, en especial para pensar la representación dicotómica de la relación entre civilización y barbarie. Sin embargo, esta nueva mirada sobre un viejo objeto de estudio no ha permeado de la misma manera en otras áreas de la historia como la dedicada al estudio de las redes. Partiendo de que la sociedad es una estructura flexible, interdependiente y en movimiento ${ }^{3}$, los estudios de redes han enfatizado el papel del actor social en su entorno más allá de los factores de clase. Esta preocupación lleva a considerarlo como parte de un sistema relacional que busca comprender el impacto de la circulación "de bienes y servicios tanto materiales como inmateriales entre sus miembros"4. Aunque las reflexiones

Nación para crear uno de carácter trasnacional. María Victoria Crespo, "Polisemia y política del concepto de frontera", en En torno a fronteras intelectuales. Conceptualizaciones, itinerario y coyunturas institucionales, comps. Horacio Crespo, Luis Gerardo Morales y Alejandra Navarro Mina (México: Editorial Itaca, Universidad Autónoma del Estado de Morelos, 2014), 17, 18, 21, 25.

3 Al respecto, Norbert Elías plantea reflexiones importantes al mencionar el peso de este contexto relacional, que traduce como las cadenas invisibles que llevan todos los que deambulan por la calle "aparentemente ajenos e independientes de los demás". Esto rompe con la idea de clases porque, sin importar la jerarquía que ocupen al interior, todos son parte de ese “armazón”, pero dado que el contexto está en permanente cambio y movimiento, el contexto deja su carácter estructural rígido para tomar uno más flexible espacial, en el cual la categoría que domina la interpretación es la interdependencia y no la dependencia.Norbert Elías, La sociedad de los individuos (Barcelona: Ediciones Península,1990),.28-35.

4 Michel Bertrand, "¿Grupo, clase o red social? Herramientas y debates en torno a la reconstrucción de los modos de sociabilidad en las sociedades de antiguo régimen”, en Redes intelectuales y formación de naciones en España y América Latina 1890-1940, eds. Martha Casáus y Manuel Pérez Ledesma (México: Universidad Autónoma de México, 2005), 52. Para Michell Bertrand una red es una estructura constituida por un conjunto de puntos y líneas que permiten visualizar las relaciones mantenidas en un momento dado por un conjunto de individuos entrelazados por un sistema de intercambios de bienes o servicios. Dichos lazos son históricos tanto y en cuanto se realizan en períodos -más o menos largosmanteniéndose o desapareciendo de manera temporal -hasta reactivarse-, o de manera definitiva. Esta variable histórica permite pensarla también cómo cambia de contenido y varía su intensidad. Michel Bertrand, "Del actor a la red: análisis de redes e interdisciplinaridad”, Nuevo Mundo Mundos Nuevos. Colloques (2009): 13, 14, http://nuevomundo.revues. org/57505; doi : 10.4000/nuevomundo.57505., 
teóricas y metodológicas han avanzado a partir de la suma de estudios de caso, y de que todos ellos se analizan en un tiempo y lugar determinados de la historia, el debate en torno al significado del espacio aún no es importante ${ }^{5}$. El descuido es mayor al tratarse de redes intelectuales en las cuales se observa la interacción de sujetos específicos que se vinculan en función del intercambio de capitales simbólicos y bienes culturales ${ }^{6}$.

En este sentido, el presente ensayo realiza un breve balance historiográfico de algunos trabajos sobre las fronteras en la historia, el papel del intelectual como sujeto de frontera y el carácter simbólico de aquellas mencionadas en estudios sobre redes intelectuales. En una segunda parte, se enfoca en una red de la década de 1920, La Unión Latino Americana durante sus primeros años de vida entre 1923 y $1925^{7}$, para reflexionar en dos coyunturas específicas:

5 Para un balance del avance en este campo remitimos a Michel Bertrand, Sandro Guzzi-Heeb y Claire Lemecier, "Introducción: ¿en qué punto se encuentra el análisis de redes en Historia?", Redes-Revista hispana para el análisis de redes sociales 21, n. ${ }^{\circ} 1$ (2011). http://www.raco.cat/ index.php/Redes

6 No existe un acuerdo sobre el concepto de redes. El que se encuentra en el texto es personal y pretende poner el acento en las características de la circulación de las ideas porque consideramos que esto especifica un poco más lo planteado por Devés-Valdés, quien define las redes intelectuales como un "conjunto de personas ocupadas en la producción y difusión del conocimiento, que se comunican en razón de su actividad profesional, a lo largo de los años".Eduardo Devés-Valdés. Redes intelectuales en América Latina. Hacia la constitución de una comunidad intelectual (Chile: Universidad de Santiago de Chile, 2007), 30.

7 Esta organización antiimperialista y latinoamericanista se desarrolló durante la década de 1920 y conformó a lo largo de sus ocho años de vida una amplia red con contactos en distintos puntos del continente americano. Su finalidad esencial era generar una opinión pública favorable a la unidad cultural, política y económica de los países de la región al intentar reflotar el viejo ideal bolivariano. Para ello, conformaron un grupo de intelectuales en torno a Renovación. Boletín de Ideas, Libros y Revistas de la América Latina, subtítulo al cual se le agregó, dos años después de su creación, el de “Órgano de la Unión Latino Americana”. El emprendimiento periodístico precedió a la fundación de la entidad unionista conformando un primer grupo-que tomó el nombre de la publicación para su identificación-desde enero de 1923 a marzo de 1925, cuando tras fundarse la Unión se transformó en su medio de difusión hasta su desaparición en 1930. Durante sus primeros años se conformó en torno a José Ingenieros y, a su muerte, la figura que lo dirigió fue Alfredo Palacios. La red contó con más de un centenar de miembros de toda América Latina, principalmente intelectuales y jóvenes que defendían la reforma universitaria -y el ideario de la nueva generación juvenilista-, se proclamaban en contra de la intromisión estadounidense en la región y en contraposición defendían la idea de una América Latina unida. Ver, Alexandra Pita, La Unión Latino Americana y el boletín Renovación. Redes intelectuales y revistas culturales en la década de 1920 (México: El Colegio de México, Universidad de Colima, 2009). 
la de su fundación, para observar cómo una nueva red se entrelaza con otras, y cuando surge una ruptura importante en ella y se separan algunos de sus miembros para formar otra red. De este modo, si en la primera la frontera es una zona porosa de libre tránsito, en la segunda se convierte en un límite infranqueable de exclusión.

Partimos de la idea inicial de que las redes no pueden ser analizadas como composiciones homogéneas, aunque se encuentren relacionadas en torno a una $o$ varias figuras intelectuales (ego o multicéntricas), ni mucho menos que son estáticas, porque se modifican de manera permanente como parte de un complejo juego de relaciones de poder. Siguiendo la idea de Pierre Bourdieu sobre campo intelectual, la cual provee la imagen de un "campo magnético", donde se encuentra un "sistema de líneas de fuerza" que se "oponen y agregan", por lo que es la distribución de este sistema compuesto por fuerzas lo que trasmite la "estructura específica" de cada campo ${ }^{8}$, pensamos que las fronteras deben ser pensadas en función del juego entre oposición y asociación, es decir, entre límites no permeables y otros que sí son porosos, y que este juego debe ser contemplado en su dimensión temporal para recuperar su dinamismo.

\section{Fronteras simbólicas e intelectuales}

Las investigaciones más recientes sobre las fronteras históricas hacen referencia a que estas se encuentran en crisis no porque hayan desaparecido, sino porque se encuentran en un proceso de redefinición como concepto. Así, al observar por qué el límite territorial de tal o cual Estado se utiliza como sinónimo de frontera a partir de la culminación de un proceso, se problematizan las fronteras como zonas de tensión y de contención, lo cual requiere de un aparato burocrático especializado y facultado para vigilar que se respete la configuración original (heredada de la colonia en el caso latinoamericano) y se organice esta territorialidad a través de políticas e instituciones públicas 9 .

8 Pierre Bourdieu, "Campo intelectual y proyecto creador", en Problemas del Estructuralismo, eds. Jean Pouillon et ál. (México: Siglo XXI, 1971), 135.

9 Juan Carlos Arriaga-Rodríguez, El largo proceso histórico de partición territorial en América Latina y el Caribe (México: Editorial Bonilla y Universidad de Quintana Roo, 2012), 15-17. Este énfasis pone de manifiesto la redefinición de una tradición en los estudios históricos sobre fronteras, la que se logra ubicar a fines del siglo XIX con el libro del historiador norteamericano Frederick J. Turner, The Significans of theFfrontier in American History (1894). En él interpretó la historia de su país a partir de la existencia del oeste como territorio de frontera ganado a través de un proceso que transformó política, económica y socialmente la nación de una manera positiva. Esta interpretación generó una tradición de estudios de 
Ahora bien, desde el estudio de las prácticas religiosas se plantea que las fronteras simbólicas son múltiples al entrar en contacto zonas de cruce (con sus correspondientes "zonas fronterizas", "de contacto", "espacios intersticiales" o "procesos de interfaz". Esto hace que imaginariamente pueda dividirse la territorialidad por las que transcurren estas prácticas religiosas entre el centro y el margen que gravita a su alrededor, espacios vinculados al permitir "cruces y contactos, tránsitos de diversos elementos”. De este modo, las fronteras operan como estructuras emergentes y contingentes más que predeterminadas. Así, el concepto de frontera se encuentra implícito en la noción de cultura al concebirse como un conjunto humano delimitado con precisión por sus costumbres, creencias y prácticas, y este, a su vez, supone posiciones y relaciones de elementos dentro de una estructura abstracta que comparte un territorio funcional entre sus integrantes para marcar la diferencia con otras culturas. Al pensar de este modo la cultura es posible concebir una comunidad cultural no desde una territorialidad física, sino como un "espacio subjetivo, incalculable y no lineal"10.

frontera en aquel país, la cual mantuvo y alimentó el interés en esta área temática. Aunque con el paso de los años la corriente se dividió entre quienes discutían abiertamente los principios turnerianos y quienes los reinterpretaban buscando una explicación menos mecanicista, sostuvo un interés creciente y constante en los estudios históricos de la frontera. El proceso que siguieron estos estudios en América Latina fue distinto. A fines del siglo XIX y principios del XX el estudio de la frontera no fue tomado en cuenta por las historias nacionales, siendo recuperado por la literatura y los relatos de viajeros. Unas y otras versiones alimentaban la percepción de una frontera en un sentido negativo, como el espacio donde la civilización había fracasado y reinaba la barbarie. Sin embargo, hacia la década de 1970 hubo un renacimiento del debate sobre la frontera en la historiografía latinoamericana, en especial en el Río de la Plata, influenciado por una reorientación de los estudios en Norteamérica. A partir de ello, se tomaron en cuenta los estudios locales y regionales realizados desde la perspectiva de la historia rural y los estudios interétnicos, que aportaron una nueva mirada sobre la frontera en la que confluyen conceptos y métodos de la historia y apropiaciones de otras disciplinas como la sociología, la economía y la antropología.Robert Schmit, "La construcción de la frontera decimonónica en la historiografía rioplatense”, Mundo agrario 8, 16 (primer semestre 2008):1, 2, 4, 10, 11, doi: http://www. redalyc.org. articulo.oa?id=84581606.

${ }^{10}$ Los autores señalan específicamente que el espacio que se genera de este entrecruzamiento, a modo de una tipología intersticial, es el que permite observar los "elementos heterogéneos y descentrados en estado de desplazamiento o en movimiento.., Daniel Solís Domínguez y Consuelo Patricia Martínez Lozano, "Construcción de fronteras simbólicas y prácticas religiosas”, Estudios fronterizos, nueva época 13, 25 (enero-junio de 2012): 10-12, doi: http:// www.redalyc.org/articulo.oa?id=53023332001. 
Esto supone utilizar la idea de espacio cultural a partir de la noción del espacio general - entendido como territorio con límites- para asimilarlo al núcleo de una identidad colectiva que despliega estrategias para delimitar el perímetro de "lo que contiene y es contenido por un contexto". Siguiendo esta idea, el contraste con lo opuesto (entendido en clave de aquello que queda fuera de mi territorialidad espacial) es esencial para entender el sentido de pertenencia de los integrantes de esa territorialidad, con sus respectivas alianzas y confrontaciones en el tiempo ${ }^{11}$.

El sentido de pertenencia y la necesidad de límites con lo externo para delimitar la identidad de un grupo pueden ser útiles para pensar el carácter del intelectual como un sujeto social que participa de esta creación de fronteras. Si consideramos el concepto de intelectual tanto en su sentido de inteligentzia (asociado a la elite de la ilustración) como de intelectual comprometido "engagés" ${ }^{2}$, se advierte una delimitación inicial: existe un grupo de personas que comienzan a ser identificadas (y a autoidentificarse en busca de su legitimación) por el manejo que tienen sobre el conocimiento y la capacidad de sostener su capital cultural. De este modo, algunos autores enfatizan que los intelectuales son sujetos de frontera frente a otros poderes y campos tradicionales (político y económico) y enfatizan la necesidad de observar las diferencias de las redes a partir de su vinculación con el poder político. Para Estrella, este tipo de red serviría como medio para introducir formas de autoridad exógenas a la hora de dirimir las pugnas estrictamente intelectuales, lo cual define, desde la perspectiva de Pierre Bourdieu, un espacio de conflicto y tensiones, pero también encuentros y alianzas entre redes contiguas ${ }^{13}$.

Esto permite pensar la posición de los intelectuales dentro del espacio cultural y cobra un especial significado la mirada sobre aquellos que pueden servir de conectores al estar ubicados en las líneas divisorias. Esta idea ha permitido a algunos autores dirigir sus investigaciones hacia el análisis de

${ }^{11}$ Teresita Quiroz. "Reflexiones sobre el espacio. A manera de prólogo”, en El espacio. Presenciay representación, coord. Leonardo Martínez Carrizales y Teresita Quiroz Ávila (México: UAM Azcapotzalco, 2009), 24, 25.

${ }^{12}$ Se incluye en él a todos aquellos que "han adquirido, con el ejercicio de la cultura, una autoridad y un influjo en las discusiones públicas". Carlo Marletti, "Intelectuales", en Diccionario de política, eds. Norberto Bobbio, Nicola Mattetucci y Gianfranco Pasquino (México: Siglo XXI Editores, 2002), 820.

${ }^{13}$ Alejandro Estrella González, "La filosofía mexicana durante el régimen liberal. Redes intelectuales y equilibrios políticos" Signos Filosóficos XII, 23 (enero-junio, 2010): 141, 144,. doi: http://www.redalyc.org/articulo.oa?id=34316039006. 
la circulación de ideas (científicas, artísticas) y, con ello, al intercambio de bienes culturales a un nivel internacional no como una "influencia de un escenario nacional o cultural sobre otro", sino como redes nutridas por la circulación de libros y revistas, viajes, conferencias, congresos y todo aquello que permita la difusión del conocimiento ${ }^{14}$. Otra manera de analizar el carácter trasnacional es entender a los intelectuales de frontera como aquellos que actúan superando no solo una condición territorial, sino que, además, poseen la capacidad de "escandir las disputas sobre los límites entre distintos tipos de saberes y discursos ${ }^{15}$.Así, la frontera sería una especie de "condensadora de un determinado tipo de experiencia intelectual "a través del cual puede observarse la aparición de pensamientos antes marginales en los horizontes historiográficos que en un momento determinado se convierten en ideas centrales para determinados campos del conocimiento"16.

En este sentido, Claudio Maíz observa la necesidad de referirse a nuevas fronteras en el campo intelectual para superar el énfasis geográfico y remitirse más al ámbito simbólico que construyen los intelectuales. Al flexibilizar las formas de observar la construcción de territorialidad puede analizarse el efecto de las políticas editoriales como espacio simbólico y como creadoras de fronteras; así se muestra hasta dónde es posible hablar de una "autonomía e independencia" entre esta y aquellas fronteras que se derivan de lo que Pascale Casanova denominó "la República Mundial de las Letras" ${ }^{17}$. Esto significa que se articulan entre sí para conformar lo que Devés llama "espacios metanacionales (más allá del Estado-nación)" los cuales tienen como atributo poder articular espacios periféricos con centrales. Sin predeterminar la geografía humana de estas redes a estructuras rígidas, afirma que estas redes interactúan como agentes desde distintos lugares para alcanzar un lugar en el ámbito internacional y, para ello, es necesario comprender los centros culturales de conexión entre

${ }^{14}$ Christophe Charle, Jürgen Schriewer y Peter Wagner (comps.), Redes intelectuales trasnacionales. Formas de conocimiento académico y búsqueda de identidades culturales (Barcelona, México: Ediciones Pomares, 2006), 9.

${ }^{15}$ Andrés Kozel. "Las marcas de heterodoxia en los historicistas mexicanos", en Heterodoxia y fronteras en América Latina, eds. por Andrés Kozel, Horacio Crespo y Héctor A. Palma (coord.) (Buenos Aires: Editorial Teseo, 2013), 164.

${ }^{16}$ Navarro, 2013, p. 147.

${ }^{17}$ Claudio Maíz, "Fronteras trasnacionales. La modernización literaria en dos momentos (1900-1920 y 1960)", Cuadernos del CILHA (Universidad Nacional de Cuyo) 7, 7-8 (2005): $292,293,300$. 
los miembros de las redes dentro y fuera del espacio periférico ${ }^{18}$. Tal parece que es ese sentido de periferia lo que promueve la creación de redes.

El caso latinoamericano da muestras de numerosos intentos de los intelectuales por superar los límites espaciales de los Estados nacionales. Esto es observable a través de la creación de numerosas redes trasnacionales que les permiten configurar otra pertenencia territorial donde se desdibujan las nacionalidades para adquirir un sentido internacional, asociado o legitimado en ocasiones con el humanismo como pensamiento homogeneizante. En otros casos, estos discursos hacen alusión a una "gran patria", "federación”, o "unión" (latinoamericana), espacio imaginario que se perdió tras las independencias del siglo XIX al crear una veintena de naciones independientes. En tono nostálgico o con sentido pragmático, las alusiones a esta otra territorialidad histórica se convierten en una práctica discursiva que alimenta los proyectos de integración regional. Esta idea hace comprensible que el ideario de la Unión Latino Americana y del Boletín Renovación fuera crear una conciencia favorable en la opinión pública con el fin de que esta presionara a sus gobiernos a tomar las medidas necesarias para formar una confederación entre los países de América Latina que contrarrestara el poderío estadounidense en la región. $\mathrm{Al}$ ser los intelectuales los encargados de crear una patria latinoamericana, debían delimitar discursivamente una geografía imaginaria excluyente de la otroriedad a la que se oponían, e incluyente de aquellos otros pares a los que llamaban a realizar una misión cultural. Por ello no es extraño que su red fuera trasnacional ${ }^{19}$.

Por su parte, Ramiro Zó advierte que las redes intelectuales en las que participa el sujeto de estudio es un espacio que posee "una naturaleza dinámica, vacilante, elástica y porosa que interconecta puntos distantes entre sí y articula un territorio cultural con fronteras que sobrepasan las de la nación”. Por ello, las dimensiones de esta territorialidad tienen un carácter "trasnacional y

\footnotetext{
${ }^{18}$ El autor menciona para el caso latinoamericano la década de 1920, las ciudades de Madrid y París y los intelectuales del centro que contribuyeron a formar redes periféricas como Henry Barbusse, Annie Besant y Willi Muzenberg. Eduardo Devés-Valdés, "La intelectualidad periférica entre 1920 y 1940: un intento de una cartografía y de un planteamiento teórico". Cuadernos del CILHA 12, 14 (2011): 93, 103, URL: http://www.redalyc.org/articulo. oa?id=181721529005.

${ }^{19}$ Pita, La Union Latino Americana.
} 
policéntrico”, el cual depende de los viajes y movimientos que realicen los miembros de la red ${ }^{20}$.

Para la formación de este espacio es indispensable estudiar los ámbitos de sociabilidad intelectual por los que circulaban las ideas, como es el caso de ateneos, centros, institutos, y universidades, en general ${ }^{21}$, y aquellos temporales como conferencias, homenajes, coloquios y cursos. A este gran número de posibilidades agregamos las revistas culturales porque consideramos que tienen una riqueza singular para el análisis de los intelectuales y sus redes. En las revistas culturales, como lugares y contextos de enunciación, puede verse cómo los intelectuales cumplen con la difusión de las ideas sumando la labor de editores, autores, lectores, críticos y comités editoriales ${ }^{22}$. De hecho, las revistas fueron un espacio privilegiado para intelectuales exiliados por la inestabilidad política de sus países, por lo que sirven de termómetro para medir la creación y transformación de redes ${ }^{23}$. Como ha señalado Ricardo Melgar en varios trabajos dedicados a los exiliados apristas, es indispensable valorar el papel que jugaron las publicaciones en las redes intelectuales como espacios de sociabilidad, pero también como emprendimientos políticos y culturales ${ }^{24}$. Por ello, algunos investigadores los consideran "enclaves" de una geografía virtual, que al correr de los años y cambiar de contextos puede incluso terminar modificando aquel sentido de frontera trasnacional ${ }^{25}$.

${ }^{20}$ Ramiro Esteban Zó, "La obra de Alfredo Bryce Echenique desde la óptica de las redes intelectuales-literarias", Cuadernos del CILHA 12, 14 (2011): 204, URL: http://www.redalyc.org/ articulo.oa?id=181721529011.

${ }^{21}$ De este tipo de espacios de sociabilidad existen trabajos que exploran la importancia de las redes académicas en la construcción de proyectos e intercambios científicos. Para analizar los vínculos entre ambos lados del Atlántico existe un especial interés en los realizados por intelectuales españoles y latinoamericanos [Ver entre otros, el estudio sobre la Junta de Ampliación de Estudios e Investigaciones Científicas, JAE, de Consuelo Naranjo Orovio y Miguel Ángel Puig-Samper, “Las redes de la ciencia: la JAE en el exilio”, Asclepio. Revista de Historia de la Medicina y de la Ciencia LIX, 2 (julio-diciembre 2007)]., pero se han realizado también otros importantes esfuerzos por estudiarlos.

${ }^{22}$ Aimer Granados, “Introducción”, en Las revistas en la historia intelectual de América Latina: redes, política, sociedad y cultura, coord.. Aimer Granados (México: UAM, 2012),.9-10.

${ }^{23}$ Regina Crespo, "Introducción”, en Revistas en América Latina: proyectos literarios políticos y culturales, coord. Regina Crespo (México: UNAM, 2010), 13.

${ }^{24}$ Ver Melgar, 2003 y 2009.

${ }^{25}$ El autor estudia el caso de las redes intelectuales de españoles y mexicanos que se asocian a través de la Revista Moderna de México, analizándola para detectar cómo sirvió de enlace entre ambos márgenes la producción literaria y artística. Gemma Gordo Piñar, "El papel de 


\section{Oposición y asociación en una red intelectual a través del tiempo}

Lo mencionado hasta aquí puede generar la falsa impresión de que las redes intelectuales son agrupamientos homogéneos y estáticos, por lo que podría pensarse que existe un único tipo de frontera (externa) que delimita la existencia de esta red en función de la oposición a otras redes políticas y económicas. Para contrarrestar esta idea proponemos dividir el análisis en dos partes: la primera dedicada a observar el momento de fundación de una revista -el cual suele ser un aspecto visible de la formación de una red- $\mathrm{y}$ sus primeros años de vida y, en la segunda, el momento en que existe un cambio importante en la composición de una red por una división interna.

Esto implica que debemos imaginar las fronteras en las redes intelectuales a partir de este juego de tensiones entre las fuerzas de asociación y de oposición. La proximidad entre una red y otra puede deberse a que una parte de sus miembros participa en ambas redes (porque los mediadores juegan un papel destacado), a que su competencia es menor porque se complementan para enfrentarse a una red hegemónica de mayor peso, o solo porque existe temporalmente un statu quo. En cualquiera de estos casos, nos encontramos con una inexistencia de frontera entendida como límite, puesto que el grado de interconexión implica una zona fronteriza amplia y dinámica, permeable al intercambio significativo de bienes culturales y capitales simbólicos. En cambio la oposición implica una frontera infranqueable entre una red y otra, debido a que no existe un desinterés mutuo o, por el contrario, a que ambas compiten por detentar un capital simbólico similar. En ambos casos, la frontera está representando un límite difícilmente franqueable para las partes, un espacio vacío que impide el intercambio.

El esquema planteado en el apartado anterior está pensado para analizar un momento determinado, esto es, el momento fundacional, por lo que a manera de una fotografía solo capta una imagen estática. Cabe preguntarse qué pasa cuando pensamos en un tiempo más amplio de manera diacrónica y no sincrónica; es claro que su composición no se mantendría igual. Los integrantes de una red interactúan de manera permanente dentro y fuera de esta agrupación, por lo que la dimensión de una red cambia irremediablemente al modificarse su composición y los vínculos existentes entre sus integrantes. Cada modificación interna implica replantearse cuál es la posición que ocupa esa red en el campo intelectual. De este modo, las fronteras pueden modificarse

las redes intelectuales en la construcción y reconstrucción del pensamiento filosófico", Bajo Palabra. Revista de Filosofía, II Época (2012): 500. 
al disminuir los límites infranqueables y aumentar las zonas fronterizas o, al contrario, al aumentar la delimitación para diferenciarse y con ello distanciarse de otras redes.

A estas modificaciones permanentes que, de manera gradual y continua, pasan casi imperceptibles, se agregan otras menos sutiles, más drásticas y observables. Si las primeras nos remiten a una cotidianeidad relativamente pacífica en la que pueden dirimirse las diferencias, las segundas lo hacen a momentos de cambio, cuando se producen crisis de legitimidad o luchas por el poder y las diferencias terminan en separaciones.

Este último caso se relaciona generalmente con la modificación del ego en una red porque el cambio de liderazgo suele acompañarse de luchas y disputas entre fracciones internas para posicionar nuevamente a sus miembros tras el cambio. Esto puede derivar en la exclusión de algunos miembros, ya sea porque fueron expulsados o porque ellos mismos deciden irse. En ambos casos, esta división suele generar una reestructuración importante de la red original, así como la creación o fundación de otras nuevas, las cuales van a disputar con la anterior su legitimidad al interior del campo intelectual.

\section{a. Inicios y delimitaciones}

La red latinoamericanista que generó el intelectual argentino José Ingenieros tuvo su origen a inicios de la década de 1920 cuando de manera regular se estableció contacto epistolar entre el argentino y un número significativo de personalidades que se habían destacado por una crítica del intervencionismo que realizaba Estados Unidos en la región. Se trataba de políticos, diplomáticos, escritores, profesores universitarios, artistas y científicos que publicaban artículos, libros o, al menos, expresaban por correspondencia su postura. Poco después, en octubre de 1922, Ingenieros plasma estas ideas en un discurso titulado "Por la Unión Latino Americana" en el homenaje a José Vasconcelos realizado en Buenos Aires por los intelectuales argentinos; discurso que circuló rápidamente por la red preestablecida y anticipó el camino para que dos meses mas tarde apareciera el primer número de Renovación, Boletín de Ideas, Libros y Revistas de la América Latina ${ }^{26}$.

${ }^{26}$ Es de especial importancia diferenciar el origen de una red con su momento fundacional, porque se da por sentado que el primer momento de una red es cuando aparece el primer número de una publicación que es vocera de sus ideas. Alexandra Pita, "Las revistas culturales como soportes materiales, prácticas sociales y espacios de sociabilidad”, en Almacenes de un tiempo en fuga: revistas culturales en la modernidad hispánica, eds. Hanno Ehrlicher y 
La red se identificó con el nombre de la publicación como el grupo Renovación desde enero de 1923 a marzo de 1925, cuando se fundó La Unión Latino Americana, asociación que finalizó recién en septiembre de 1930. Durante los primeros años de esta red, quienes integraron el grupo fundador del Boletín fueron Luis Campos Aguirre (seudónimo de Aníbal Ponce), Julio Barreda Linch (seudónimo de José Ingenieros) y Gabriel S. Moreau (quien aparecía como el director de la publicación). Formalmente, los tres fundadores compartían responsabilidades, pero es evidente que existió una marcada jerarquización, pues Ingenieros era quien concentraba el mayor capital social debido a que gozaba de un amplio prestigio intelectual. Además, contaba con una extensa red de contactos nacionales e internacionales. Por esto, la red que giró en torno a la publicación tuvo como ego a Ingenieros.

Ingenieros influyó decisivamente en la definición de un discurso textual y visual para la publicación con el fin de generar una opinión pública favorable a la unidad cultural, política y económica de los países de la región al intentar reflotar el viejo ideal bolivariano. A ese ingrediente discursivo sumó una fuerte crítica al expansionismo norteamericano y se asoció entonces a una propuesta de unidad regional a través de un discurso compuesto por representaciones de América Latina y una práctica que se basaba fundamentalmente en construir una amplia red intelectual ${ }^{27}$.

Esto supone que la territorialidad imaginaria en la que se suponía estaba la red era fuertemente influenciada por el sentido de oposición al imperialismo, convirtiéndose en un límite no franqueable que señalaba un sentido de pertenencia para los integrantes, así como sus posibles alianzas con otras redes y publicaciones que comulgaran con este principio. No es

Nanette Rißler-Pipka (Aachen: Shaker Verlag, 2014), 236, 237. Sobre esta red y la difusión del discurso remitimos a Alexandra Pita, "La difusión de un discurso latinoamericanista y la creación de una red intelectual, 1922-1924, en Redes intelectuales trasnacionales en América Latina durante la entreguerra, comp. Alexandra Pita (México: Editorial Miguel A. Porrúa, Universidad de Colima, 2016).

${ }^{27}$ Aunque la red se declara en contra de todos los imperialismos extranjeros, puntualizan el estadounidense por ser este el que representa mayor peligro al dedicarse a enemistar a los latinoamericanos con el fin de convertirlo en enemigos y de esa manera debilitarlos para intervenirlos. Para contrapesar ese poderío proponían crear una conciencia latinoamericana basada en la cooperación y la solidaridad, no como el viejo plan político de una confederación porque los gobiernos estaban subordinados a la voluntad estadounidense., Editorial “Fijando Rumbos", Renovación 1, 3 (marzo 1923); Editorial "Federación”, Renovación 2, 4 (abril 1924): 1. 
extraño entonces que los colaboradores que sumaron su participación al Boletín aludieran permanentemente a una "gran patria", "federación", o "unión" (latinoamericana), como un espacio que se perdió tras las independencias del siglo XIX al crearse fronteras internas que generaron una veintena de naciones independientes. El uso de estos términos va asociado en repetidas ocasiones por una representación de continente con una territorialidad que abarca desde el río Bravo -al norte- hasta el estrecho de Magallanes -al sur ${ }^{28}$.

Esto generaba que la red en torno a Renovación tuviera un límite con otras publicaciones culturales que no tenían un perfil político similar, pero hacía posible que se acercara a otras con las que mantenía canje y publicidad. Entre estas se encontraron las revistas Valoraciones y Sagitario, ambas integradas por estudiantes, graduados y profesores de la Universidad de La Plata que se decían pertenecientes a la "izquierda reformista”. Agrupados inicialmente en torno al periódico de la Federación Universitaria Platense titulado (también) Renovación, este grupo se había dedicado fundamentalmente a dar manifestaciones públicas teatrales, actuando ocasionalmente en la política universitaria de la Facultad de Humanidades en apoyo a la candidatura de Alejandro Korn. En julio de 1923 el grupo se consolidó en torno a la revista Valoraciones, dirigida por Carlos Américo Amaya que apareció gracias al apoyo de Korn y Arnaldo Orfila Reynal, pero al poco tiempo, (en enero de 1925), hubo una divergencia interna que ocasionó el cambio de dirección de la revista cuando Amaya renunció, dejando en su cargo al propio Korn, para fundar Sagitario junto a Carlos Sánchez Viamonte y Julio V. González ${ }^{29}$.

Aunque Renovación era más combativa por su carácter antiimperialista y latinoamericanista, compartía con las revistas platenses ser órganos de expresión de la nueva generación americana, entre cuyos múltiples ideales de cambio se encontraba también -aunque más atenuado-, el ideal integracionista. No es extraño entonces que Sánchez Viamonte, González y Amaya fueran colaboradores asiduos del Boletín desde su inicio, ni que participaran en marzo de 1925 en la fundación de la Unión Latino Americana: Sánchez Viamonte se convirtió en el vicepresidente de la Unión Latino Americana (de 1925 a

\footnotetext{
${ }^{28}$ América Latina es considerado un continente compuesto de varias nacionalidades comunes asociadas porque sus pueblos tienen "análogos orígenes, desenvolvimiento y porvenir". Esta representación está delimitada desde el primer editorial y no se modificó a través de sus ocho años de vida. Editorial “Renovación”, Renovación 1, 1 (enero 1923): 1.

${ }^{29}$ Luis Aznar, Universidad nueva y ámbitos culturales (La Plata: Universidad de la Plata, 1963), $247-254$
} 
1930), González fue miembro del Consejo, mientras Amaya se mantuvo como adherente y colaborador del Boletín. Además, cumplieron un importante rol dentro de la red unionista: ser mediadores culturales al pertenecer a ambas redes y permitir a través de esto una mayor circulación de ideas.

Caso similar ocurrió con la revista Inicial fundada por jóvenes de la nueva generación que lanzaron su publicación en octubre de 1923 en Buenos Aires. Entre sus fundadores se encontraba Alfredo Brandan Caraffa, quien participó también de la fundación de la Unión Latino Americana en marzo de 1925. La red unionista reproduce "complacida" en sus páginas el programa inicial porque coinciden en varios aspectos discursivos: su rechazo a todo lo anterior a la Primera Guerra Mundial (a quienes tachan de decadentes e inmorales), su exaltación de la juventud como fuerza moral de la humanidad ante los problemas contemporáneos, su rechazo a ser una más de las "desteñidas" publicaciones del ambiente artístico y literario, "una antología pálida e inmóvil de los poetas y escritores jóvenes del país" 30 .

De este modo, estas publicaciones formaban una red de revistas que pretendían representar a la nueva generación, aquella menor de los 30 años que, pese a sus diferencias ideológicas y artísticas, tuvieran puentes que las conectaran a través de esta empatía generacional, no en un sentido biológico del término, sino de pertenencia e identificación de ciertos valores. Para Fernando Diego Rodríguez esto significa que estas revistas tendían a "conformar constelaciones que agrupaban emprendimientos afines por cuestiones que, si a veces se afirmaban en lo estético o ideológico, muchas otras solo pueden ser interpretadas desde una clave generacional y dentro de esta clave, por los ámbitos de circulación de los jóvenes intelectuales: la universidad, la bohemia literaria, el cenáculo, entre los más notorios" ${ }^{31}$.

$\mathrm{Al}$ morir José Ingenieros, en octubre de 1925, las tres publicaciones reaccionan. Algunas se mantuvieron dentro de los límites fronterizos de esta red de revistas en las que coincidían varios de sus miembros porque compartían una afinidad política y se oponían a su vez a otras publicaciones de perfil más literario. Este fue el caso de Sagitario y Valoraciones. La primera le dedicó un

30 "Roberto A Ortelli, Brandan Caraffa, Roberto Smith y Romero M Gugliemini fundan Inicial”, Renovación 1, 9 (octubre de 1923): 7. Esta publicación se publicitó en las páginas del Boletín y además se reprodujeron algunos artículos completos sobre la reforma universitaria y literatura. Pilades Dezeo, "El mantenimiento de la Reforma Universitaria”, Renovación 1, 9 (octubre 1923): 8; Eduardo Ripa "De la ética en la literatura", Renovación 2, 2 (febrero de 1924):6; R. S., "Elías Castelnuovo. Tinieblas”, Renovación 2, 2 (febrero de 1924): 7.

${ }^{31}$ Rodríguez, 1995, p. 62. 
número como homenaje al "Maestro" de la juventud universitaria que defendía los principios idealistas de la reforma. La segunda defendió ideas muy similares al reproducir en sus páginas el discurso que pronunció en el sepelio su director Carlos Sánchez Viamonte ${ }^{32}$. En cambio, Inicial encontró en este momento una coyuntura idónea para trazar un límite fronterizo infranqueable entre su red y la de Renovación. Por ello, uno de sus fundadores y miembro del grupo editorial, Homero Guglielimini, escribió un artículo en la revista Nosotros para cuestionar a Ingenieros como representante del positivismo y el naturalismo cientificista que debía ser superado por la nueva generación ${ }^{33}$.

\section{b. Reestructuración y crisis}

La muerte de Ingenieros tuvo un fuerte impacto en la organización de la Unión Latino Americana, en el desarrollo del Boletín como órgano de difusión, pero sobre todo en la composición de la red latinoamericanista. La Unión había sido fundada pocos meses antes en marzo de 1925. Al institucionalizarse, convirtió el Boletín en su órgano oficial. Con esta medida se pretendía ampliar a la red de Ingenieros para posicionarla en un lugar hegemónico y competitivo ante otras redes que presentaban un perfil similar en América Latina, como fue el caso del APRA, o de los intentos de conformar un Congreso de Intelectuales (con José Vasconcelos a la cabeza) de Edwin Elmore ${ }^{34}$.

Al nombrar como presidente de la misma al argentino Alfredo Palacios se pretendía incluir a un gran número de contactos establecidos en su larga trayectoria como militante del Partido Socialista y fundamentalmente como Rector de la Universidad de La Plata. Sin embargo, a nivel de redes coexistieron ambos personajes como nodos centrales porque aunque no tenía ningún cargo institucional, Ingenieros mantuvo su centralidad y para ello contó con el apoyo de Gabriel S. Moreau, quien pasó a figurar como el único director de Renovación, y de Arturo Orzábal Quintana un colaborador fundamental durante estos años del Boletín porque en cada número publicaba en la primera página extensos artículos dedicados a criticar el avance estadounidese a través de los organismos regionales como la Unión Panamericana y la recientemente creada en Europa Sociedad de Naciones. A la primera la interpelaba por ser un instrumento directo de control de la Secretaría de Estado de los Estados Unidos, que realizaba las farsas de las Conferencias Panamericanas para dar la imagen

32 Sagitario 2, V (enero-febrero 1926). Valoraciones 3, 8 (noviembre 1925): 7-8,

${ }^{33}$ Nosotros XIX, 199 (diciembre 1925): 606-619.

${ }^{34}$ Pita, La Unión Latino Americana, 144, 149. 
de que existía un acercamiento diplomático entre los países del continente. Mientras que el órgano intercontinental era fuertemente criticado porque permitió que en su acta constitutiva se incluyera una cláusula por la cual se legitimaba la Doctrina Monroe como principio para la región ${ }^{35}$. De este modo, reafirmó una y otra vez la validez de los principios expresados por Ingenieros sobre la necesidad de una unidad defensiva de los países latinoamericanos. Con ello, dibujó en el imaginario colectivo una frontera externa infranqueable.

Orzábal se dedicó de lleno a la red unionista. Fue un colabor asiduo entre 1923 y 1925 y, en marzo de ese año, ocupó el cargo de Secretario General de la organización. De hecho, Ingenieros le encargó que cuidara por él de su buen funcionamiento mientras emprendía un viaje largo que lo llevaría a Francia y a México. Orzábal emprendió varias labores en la ULA con el apoyo de Ingenieros para ejecutar su "magnífico plan", por lo cual informaba con detalle de las actividades realizadas a Ingenieros cuando este se encontraba fuera del país. En la correspondencia enviada durante este viaje, Orzábal se queja de que Palacios "carece de empuje y de iniciativas propias" y que el Consejo Directivo "no hace nada y le deja a él el peso íntegro de las tareas" ${ }^{36}$.

Durante estos meses, Orzábal dio inicio a otro emprendimiento, La Revista de Oriente, como órgano de difusión de otra red en la que participaban aquellos que se inscribían en la Asociación de Amigos de Rusia y buscaban que el gobierno argentino reconociera al de la URSS revolucionaria. Su conversión en un especialista en política internacional se debió a que, a su regreso de Europa en 1920, se vinculó a los grupos reformistas que giraban en torno a Ingenieros y este le abrió puertas para publicar artículos en su Revista de Filosofia y, poco después, en Renovación. Su participación en esta red ingenieriana le permitió establecer vinculos con otros intelectuales como los peruanos José Carlos Mariátegui y Víctor Raúl Haya de la Torre y el mexicano Diego Rivera, quienes participaron, igual que otros adherentes del unionismo, en la Revista de Oriente. Así, esta publicación se convirtió en una publicación más de la red porque compartían la crítica al capitalismo como sistema opresor y el ideal de luchar por una sociedad más justa e igualitaria. De este modo, como con las publicaciones anteriormente mencionadas, se establecieron publicidades compartidas y un sistema de canje ${ }^{37}$.

\footnotetext{
35 Sobre Orzábal en Renovación remitimos a Pita, La Unión Latino Americana.

${ }^{36}$ CEDINCI, FJI, SAA/8-4/6.2.81, carta mecanografiada de Arturo Orzábal a José Ingenieros, 1. ${ }^{\circ}$ de julio de 1925 .

37 Ávila, 2015, pp. 171, 188, 191.
} 
Así como en el caso de Inicial, Valoraciones y Sagitario, Orzábal se convirtió en un mediador con la Revista de Oriente. Y, como ellas, reaccionó ante la muerte inesperada de Ingenieros a fines de 1925. Su participación en los homenajes fue decisiva, pero no como miembro de ambas redes sino como representante de la Unión Latino Americana. La organización intentó, a través de los homenajes, capturar la representacion de Ingenieros como fundador del latinoamericanismo para enfrentarse de algún modo a otras representaciones que se realizaron en publicaciones varias en una búsqueda por apropiarse simbólicamente de su prestigio y nombrarse sus dignos herederos.

Renovación no fue la excepción y se dedicó a rendirle homenaje como un Ingenieros latinoamericanista, porque con ello intentaba mantener la cohesión de la red. De hecho, como representante de la Comisión Directiva, se delegó a Orzábal para escribir en nombre de la ULA, quien dedicó sus palabras al Maestro de las juventudes latinoamericanas por haber dedicado toda su vida al gran ideal que es el latinoamericanismo. Orzábal aprovechó su discurso para hacer un llamado a la juventud a participar en el unionismo que fue "la obra política más acabada” de Ingenieros durante sus últimos años. $\mathrm{Al}$ adherirse, sus herederos continuarían con su idea ${ }^{38}$.

Pese a esta aparente cohesión grupal, la muerte de Ingenieros agravó las diferencias previas entre Orzábal y Palacios, produciendo una fractura que determinó el abandono de su cargo -y de la organización- a inicios de 1926. Junto con Orzábal se fueron otros jóvenes adherentes que se separaron de la red al acusar a Palacios y al Consejo Directivo de falsear el sentido latinoamericanista de Ingenieros, demostrando así su incapacidad de formar una opinión pública en determinadas ideas. Además, los acusaban de que se habían acercado excesivamente al APRA (Asociación Popular Revolucionaria Americana) recientemente fundada en México por el peruano Víctor Raúl Haya de la Torre, a la que calificaban como representante de una ideología extremista. Ubicándose en el lugar de dignos herederos de Ingenieros, esta disidencia creó otra organización similar llamada Alianza Continental, la cual funcionó como una red paralela que compitió durante los años de 1926 a 1928 de manera permanente con la ULA ${ }^{39}$.

${ }^{38}$ Arturo Orzábal Quintana “Ingenieros y la Unidad de América” Renovación 3, 11 y 12 (noviembre-diciembre de 1925): 1 y 2.

${ }^{39}$ Para ver esta separación y la creación de una nueva red antiimperialista y latinoamericanista llamada Alianza Continental, que tuvo a Arturo Orzábal Quintana como egocentro, remitimos a Pita, La Unión Latino Americana, capítulo 4. 


\section{Conclusión}

Pensar las fronteras simbólicas de una red intelectual implica reflexionar en términos históricos sin dejar de lado una mirada interdisciplinar para reflexionar sobre la especificidad de lo simbólico, lo inmaterial. Definir estos límites no es una tarea sencilla. Los especialistas del tema no parten de una definición a priori de lo que es una frontera, sino que llegan a ella a partir de una búsqueda más compleja en la cual el estudio de casos particulares permite deducir ciertas conclusiones. Este avance significativo lleva aparejado empero, como problema, la inexistencia de una problemática general. En este sentido, coincido con lo que plantea Silvia Pappe al definir que la falta de un debate en torno al uso del término espacio en la historia complica la diferenciación entre el carácter metafórico (abstracto) de los espacios concretos (como las fronteras, agregaría $)^{40}$.

El presente ensayo solo es un ejemplo de una variada gama de posibilidades que merecen aún ser exploradas. Es conveniente proseguir en otros estudios analizando las representaciones de las fronteras que tuvieron los intelectuales participantes de las redes. Estos rasgos nos llevarían a confrontar lo dicho sobre los aspectos simbólicos y la manera en que su dimensión subjetiva afecta la construcción de categorías aparentemente físicas, como lo es, por ejemplo, el de la territorialidad. Así, la dimensión de una frontera indicaría, para una red dentro del campo intelectual, el límite en un sentido de tensión más que de definición; como una marca de identidad grupal de fuerte carga simbólica porque implícitamente conlleva las subjetividades de los actores.

Esto nos lleva a una última reflexión. Como señalamos, es importante detectar los momentos clave de una red, aquellos en los cuales podemos encontrar cambios significativos, porque es en este contexto de tensión cuando se reorganiza la relación entre los integrantes y esto a su vez modifica el lugar que ocupa cada integrante en la red (grado de centralidad). En el caso analizado, el primer momento que transcurre en sus primeros años de vida, se caracteriza por generar un espacio poroso y conectivo al establecer vínculos estrechos con intelectuales que pertenecen a su vez a otras revistas y sus redes. Se crea así una red de revistas en las que existe coexistencia de algunos contactos que sirven como mediadores de redes, y quienes juegan un papel fundamental para la construcción de una frontera porosa, permeable, como una verdadera zona

${ }^{40}$ Pappe, Silvia. "La problematización del espacio y el lugar social del historiador”, en El espacio. Presencia y representación, coordinado por Leonardo Martínez Carrizales y Teresita Quiroz Ávila (México: UAM Azcapotzalco, 2009), 32-33. 
de tránsito. Como hemos mencionado, los límites territoriales demarcados como jurisdicción de los Estados Nacionales construyeron un aparato burocrático especializado para crear, fortalecer y defender sus fronteras. En el caso de las redes, pareciera que esta función particular la asume el mediador de una red. Una vez analizado esto, podríamos volver al debate interdisciplinar para mantener el diálogo sobre la influencia de los sujetos de frontera en la configuración social del espacio.

En el segundo momento, que definimos como de reorganización y crisis, analizamos la coyuntura que se inicia a partir de la institucionalización de la Unión Latino Americana y termina pocos meses después de la muerte de José Ingenieros cuando una parte de los unionistas se separan para crear La Alianza Continental, red que, por sus objetivos, estaría integrada por los mismos contactos que tenía la unionista. Apuntamos también el papel de Orzábal Quintana porque es a través de él que se puede visualizar con mayor claridad la tensión entre asociación y oposición como dos fuerzas antagónicas que marcaron una frontera interna que inició como una marginalidad y terminó tras la ruptura como una frontera externa cuando se creó una nueva red.

Cabe recordar que, a diferencia de lo que sucede con las fronteras externas, las internas pueden mostrar una alta interconexión entre las partes, por lo que los límites son estrechos y móviles, dependientes de la necesidad de la red de diferenciarse u homogeneizarse a sí misma. Si las primeras nos remiten imaginariamente a un límite o perímetro territorial, las segundas son un lugar "amorfo en el que se activan los universos simbólicos de los sujetos y grupos", donde podemos observar que convive el complejo mundo de "lo irrenunciable con lo negociable" 41 .

Por último, cabe enfatizar el importante papel que cumplen los referentes de la red, es decir, aquellos que, vivos o no, cumplen con una función legitimadora y conectiva del grupo. Como vimos, la red unionista tenía como ego a Ingenieros mientras este fue su principal impulsor en los primeros años, espacio que mantuvo cuando ya se había fundado la Unión, y aún cuando Palacios ocupó el cargo de Presidente de la misma. Incluso después de su muerte, Ingenieros continuó siendo un nodo importante a través de los numerosos homenajes y citas, manteniendo su capacidad de generar fronteras.

${ }^{41}$ Cynthia Pech Salvador, Marta Rizo García y Vivian Romeu Aldaya. "El hábitus y la intersubjetividad como conceptos clave para la comprensión de las fronteras internas. Un acercamiento desde las propuestas teóricas de Bourdieu y Schütz”. Frontera Norte 21, 41 (enero-junio de 2009): 33,34 y 43. 


\section{Referencias bibliográficas}

Aznar, Luis. Universidad nueva y ámbitos culturales. La Plata: Universidad de la Plata, 1963.

Arriaga-Rodríguez, Juan Carlos. "El concepto frontera en la geografía humana”. Perspectiva Geográfica 17, (enero-diciembrede 2012): 71-97.

Arriaga-Rodríguez, Juan Carlos. El largo proceso histórico de partición territorial en América Latina y el Caribe. México: Editorial Bonilla y Universidad de Quintana Roo, 2012.

Bertrand, Michel. “¿Grupo, clase o red social? Herramientas y debates en torno a la reconstrucción de los modos de sociabilidad en las sociedades de antiguo régimen". En Redes intelectuales y formación de naciones en España y América Latina 1890-1940, editado por Martha Casáus y Manuel Pérez Ledesma, 47-63. México: Universidad Autónoma de México, 2005.

Bertrand, Michel, Sandro Guzzi-Heeb y Claire Lemecier. "Introducción: ¿en qué punto se encuentra el análisis de redes en Historia?" En Redes. Revista hispana para el análisis de redes sociales 21, n. ${ }^{\circ} 1$, (diciembre 2011), 1-23.

Bertrand, Michel. "Del actor a la red: análisis de redes e interdisciplinaridad”. Nuevo Mundo Mundos Nuevos. Colloques. (2009): 13, 14, URL: http://nuevomundo.revues. org/57505; doi : 10.4000/nuevomundo.57505.

Bourdieu, Pierre. "Campo intelectual y proyecto creador”. En Problemas del Estructuralismo. Editado por Jean Pouillon, et ál. México: Siglo XXI, 1971.

Charle, Christophe; Jürgen Schriewer y Peter Wagner (comps.) Redes intelectuales trasnacionales. Formas de conocimiento académico y búsqueda de identidades culturales. Barcelona, México: Ediciones Pomares, 2006.

Crespo, María Victoria. "Polisemia y política del concepto de frontera". En torno a fronteras intelectuales. Conceptualizaciones, itinerario y coyunturas institucionales". Compilado por Horacio Crespo, Luis Gerardo Morales y Alejandra Navarro Mina. México: Editorial Itaca, Universidad Autónoma del Estado de Morelos, 2014, 15-28.

Crespo, Regina. “Introducción”. En Revistas en América Latina: proyectos literarios políticos y culturales. Coordinado por Regina Crespo. México: UNAM, 2010.

Devés-Valdés, Eduardo. "La intelectualidad periférica entre 1920 y 1940: un intento de una cartografía y de un planteamiento teórico". Cuadernos del CILHA 12, n. . 14, (2011): 92-108. URL http://www.redalyc.org/articulo.oa?id=181721529005

Devés-Valdés, Eduardo. Redes intelectuales en América Latina Hacia la constitución de una comunidad intelectual. Chile: Universidad de Santiago de Chile, 2007.

Estrella González, Alejandro. "La filosofía mexicana durante el régimen liberal. Redes intelectuales y equilibrios políticos". Signos Filosóficos XII, n. . . 23, (enero-junio, 2010): 141-181. URL: http://www.redalyc.org/articulo.oa?id=34316039006

Elías, Norbert. La sociedad de los individuos. Barcelona: Ediciones Península, 1990.

Gordo Piñar, Gemma. "El papel de las redes intelectuales en la construcción y reconstrucción del pensamiento filosófico”. Bajo Palabra.. Revista de Filosofía, II Época, 2012, 495-503. 
Granados, Aimer. "Introducción”. En Las revistas en la historia intelectual de América Latina: redes, política, sociedad y cultura. Coordinado por Aimer Granados. México: UAM, 2012.

Grimson, Alejandro, "Disputas sobre las fronteras". En Teoría de la frontera. Los límites de la política cultural. Editado por Scott Michaelsen y David Johnson. Barcelona: Gedisa, 2003.

Kozel, Andrés. "Las marcas de heterodoxia en los historicistas mexicanos”. En Heterodoxia y fronteras en América Latina. Editado por Andrés Kozel, Horacio Crespo y Héctor A. Palma (coords.). Buenos. Aires: Editorial Teseo, 2013, 163-182.

Maíz, Claudio. "”. En Cuadernos del CILHA Universidad Nacional de Cuyo, Argentina, 7, n. ${ }^{\circ}$ 7-8, (2005): 287-306.. URL http://www.redalyc.org/articulo. oa?id=181720523015

Marletti, Carlo, "Intelectuales”. En Diccionario de Política. Editado por Norberto Bobbio, Nicola Mattetucci y Gianfranco Pasquino. México: Siglo XXI Editores, 2002,819-824.

Melgar Bao, Ricardo. Redes e imaginario del exilio en México y América Latina, 19341940. Buenos Aires: Ediciones Libros en Red, 2003.

Melgar Bao, Ricardo. "Comiternismo intelectual: Representaciones, redes y prácticas político-culturales en América Central, 1921-1933”. En Revista Complutense de Historia de América, 35, (2009): 135-159.

Naranjo Orovio, Consuelo y Miguel Ángel Puig-Samper. "Las redes de la Ciencia: la JAE en el exilio”. En Asclepio. Revista de Historia de la Medicina y de la Ciencia, LIX, n, ${ }^{2}$. (julio-diciembre 2007): 231-254.

Pappe, Silvia. "La problematización del espacio y el lugar social del historiador". En El espacio.Presencia y representación. Coordinado por Leonardo Martínez Carrizales y Teresita Quiroz Ávila. México: UAM Azcapotzalco, 2009, 29-54.

Pech Salvador Cynthia, Marta Rizo García y Vivian Romeu Aldaya. "El hábitus y la intersubjetividad como conceptos clave para la comprensión de las fronteras internas. Un acercamiento desde las propuestas teóricas de Bourdieu y Schütz". En Frontera Norte 21, n. ${ }^{\circ}$ 41, (enero-junio de 2009).

Pita, Alexandra. La Unión Latino Americana y el Boletín Renovación. Redesintelectuales y revistas culturales en la década de 1920. México: El Colegio de México, Universidad de Colima, 2009.

Pita, Alexandra. "Las revistas culturales como soportes materiales, prácticas sociales y espacios de sociabilidad”. En Almacenes de un tiempo en fuga: Revistas culturales en la modernidad hispánica. Editado por Hanno Ehrlicher, Nanette Rißler-Pipka. Aachen: Shaker Verlag, 2014, 227-245.

Pita, Alexandra. "La difusión de un discurso latinoamericanista y la creación de una red intelectual, 1922-1924”. En Redesintelectuales trasnacionales en América Latina durante la entreguerra. Compilado por Alexandra Pita. México: Editorial Miguel A. Porrúa, Universidad de Colima, 2016.

Pita, Alexandra. "Los homenajes a José Ingenieros y el debate en torno al papel del intelectual”. En Revista Complutense de Historia de América 35, 69-85, (2009). 
Quiroz, Teresita. "Reflexiones sobre el espacio. A manera de prólogo”. En El espacio. Presencia y representación. Coordinado por Leonardo Martínez Carrizales y Teresita Quiroz Ávila. México: UAM Azcapotzalco, 2009, 23 a 28.

Rodríguez, Fernando. "Inicial, vanguardia y reforma universitaria”. En Pensamiento Universitario 3, n. ${ }^{\circ}$ 3, (abril 1995): 62-66.

Schmit, Robert. "La construcción de la frontera decimonónica en la historiografía rioplatense". En Mundo agrario 8, n. ${ }^{\circ} 16$, (primer semestre 2008):1-17, URL: http:// www.redalyc.org.articulo.oa?id=84581606

Solís Domínguez, Daniel y, Consuelo Patricia Martínez Lozano. "Construcción de fronteras simbólicas y prácticas religiosas”. En Estudios fronterizos, nueva época 13, n. ${ }^{\circ}$ 25, (enero-junio de 2012): 9-30. URL: http://www.redalyc.org/articulo. oa? id $=53023332001$

Zó, Ramiro Esteban. "La obra de Alfredo Bryce Echenique desde la óptica de las redes intelectuales-literarias”. En Cuadernos del CILHA 12, n. . . 14, (2011): 202-208. URL: http://www.redalyc.org/articulo.oa?id=181721529011 